\title{
Krebsprävention durch Ernährung
}

\author{
Claus Leitzmann
}

\section{Zusammenfassung}

Zur Senkung des Risikos von Krebskrankheiten werden neben ausreichender körperlicher Aktivität die Vermeidung von Tabak und Übergewicht empfohlen. Entscheidend ist eine nährstoffreiche, energiebegrenzte, d.h. überwiegend pflanzliche Kost. Der Konsum von rotem Fleisch, Alkohol und Salz ist zu begrenzen, Supplemente sind nicht erforderlich.

\section{Schlüsselwörter}

Krebsprävention, Übergewicht, pflanzliche Kost, rotes Fleisch, Nahrungsergänzungsmittel

\section{Vorbemerkung}

In den letzten Jahren ist eine Flut von Veröffentlichungen über den Zusammenhang von Ernährung und Krebsprävention erschienen, aber die bisherigen Empfehlungen zur Senkung des Krebsrisikos haben sich dadurch kaum geändert. Allerdings können die Empfehlungen aufgrund der neuen Erkenntnisse ergänzt und auf der Basis sich bestätigender Erfahrungen erweitert werden.

\section{Anzahl der Krebskranken und Erkrankungsmuster}

Mit zunehmender Lebenserwartung steigt die Zahl der Krebserkrankungen und Krebstoten. Deshalb und durch die verbesserten Optionen der Früherkennung steigt die prozentuale Krebsrate einer älter werdenden Bevölkerung an. Bei Männern ist der Lungenkrebs weiterhin die führende Krebstodesursache, bei Frauen ist es der Brustkrebs. Magenkrebs geht seit Jahrzehnten bei uns deutlich zurück. Nur in Ländern wie Portugal, Chile und Japan mit einem hohen Salz-

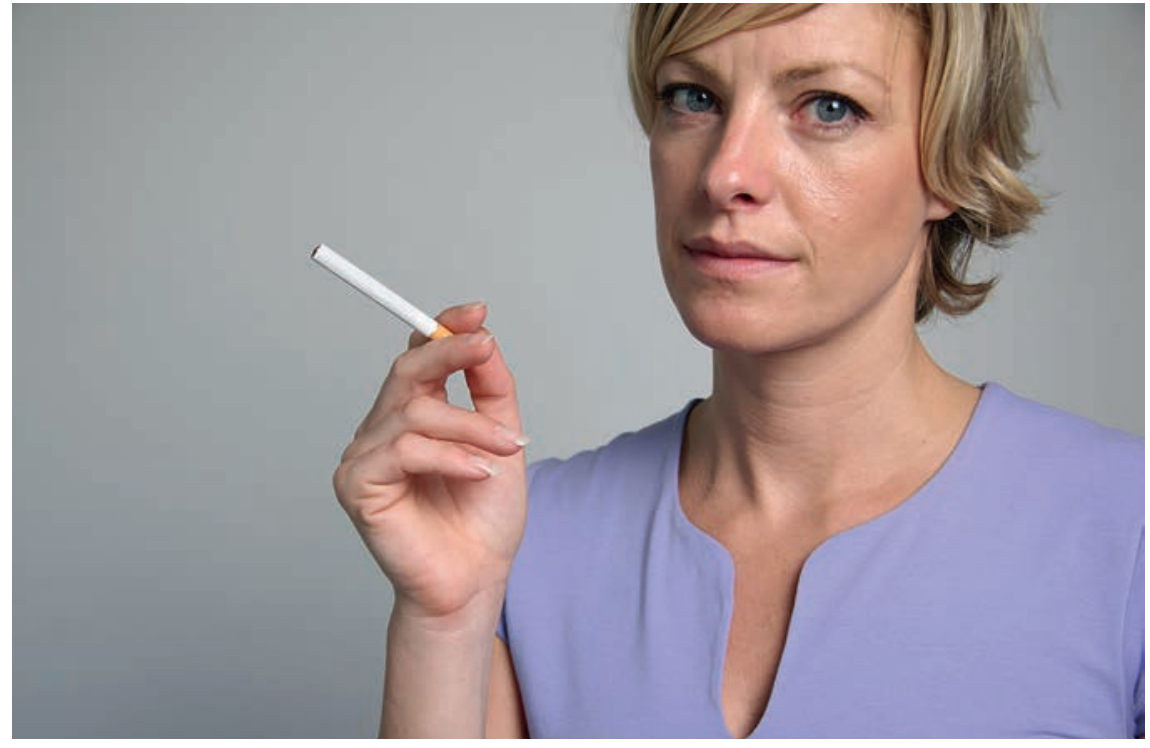

- Abb. 1 Selten sind in der Onkologie die Zusammenhänge so eindeutig wie beim Rauchen aktuell abzulesen an der steigenden Lungenkrebsrate der Frauen. ( ) Thieme Verlagsgruppe/ Christoph Frick

konsum, überwiegend durch gesalzenen Fisch, bleibt die Magenkrebsrate hoch. Lungenkrebs nimmt bei Frauen seit Jahren stetig zu und hat bei den unter Vierzigjährigen bereits das Niveau der Männer erreicht ( $\vee$ Abb. 1).

Weltweit erkranken jährlich etwa 12 Mio. Menschen an Krebs, von denen mehr als die Hälfte an dieser Krankheit sterben. Bei Nutzung der Möglichkeiten zur Krebsprävention wäre eine Reduzierung der Krebstoten um mindestens die Hälfte möglich. Deutschland liegt im internationalen Vergleich bei der Krebshäufigkeit in Industrieländern im oberen Bereich. In wirtschaftlich armen Ländern spielen die bei uns häufigsten Tumoren an Darm, Brust und Prostata eine untergeordnete Rolle, dafür finden sich häufiger Krebserkrankungen des Magens und der Leber. Diese Krebsfälle sind vornehmlich auf fehlende Möglichkeiten einer hygienisch einwandfreien Lagerung von Lebensmitteln zurückzuführen.

Die Krebsinzidenz in Deutschland liegt jährlich bei fast einer halben Million, sie ist bei Männern etwas häufiger als bei Frauen. Männer leiden am häufigsten an Krebs von Prostata, Darm und Lunge, bei Frauen sind es Brust, Darm, Gebärmutter und Lunge. Bei Frauen folgt die Krebsmortalität der Inzidenz, bei Männern ist es Krebs an Lunge, Darm und Prostata.

In Deutschland steigen die absoluten Zahlen für Neuerkrankungen im Vergleich zu den Vorjahren. So sind die Krebsformen, für die es in Deutschland systematische Früherkennungsmaßnahmen gibt, seit der Einführung dieser Untersuchungsprogramme häufiger diagnostiziert worden (z.B. Krebs an Brust, Darm und Haut). 


\section{Der WCRF-Report zur Krebsprävention}

1997 veröffentlichte der World Cancer Research Fund eine umfassende Auswertung aller Veröffentlichungen zum Thema Krebs und Ernährung. Die 2. Auflage aus dem Jahr 2007 wurde durch die Themen Übergewicht und körperliche Aktivität erweitert $(14,15)$. Seit der Zeit erscheinen jährlich Aktualisierungen des Reports zu den verschiedenen Krebsformen. Auf der Basis der gewonnenen Erkenntnisse formulierten die Experten 10 Empfehlungen zu Krebsprävention.

Empfehlung 1:

So schlank wie möglich bleiben.

Das Körpergewicht sollte während der Kindheit und im Jugendalter im unteren Bereich des normalen BMI und für $\mathrm{Er}$ wachsene innerhalb des normalen Bereichs liegen (BMI 21-23). Da eine Körpergewichtszunahme mit zunehmendem Alter nachteilig ist, wird empfohlen, eine Zunahme des Körpergewichts und des Bauchumfangs im Erwachsenenalter zu vermeiden. Die lebenslange Beibehaltung eines normalen Körpergewichts könnte eine der wichtigsten Maßnahmen zum Schutz vor Krebserkrankungen sein. Normales Körpergewicht schützt außerdem gegen eine Reihe anderer häufig auftretender, chronischer Erkrankungen.

Übergewicht ist besonders stark assoziiert mit einem erhöhten Krebsrisiko für Darm, Bauchspeicheldrüse, Brust und Nieren. Ein Übergewicht von 20-30\% gilt bereits als Risiko. Bei einer Kombination von Rauchen und Alkoholkonsum steigt das Risiko schon ab 15\% Übergewicht.

Eine Zunahme des Körpergewichts mit zunehmendem Alter gilt als normal und unproblematisch, wenn es sich in Grenzen hält und kann sogar einen gewissen Schutz gegen Krebs und Schlaganfälle im Alter bieten (3). Die viszerale Adipositas (Apfelform) und die damit einhergehende Entwicklung einer Insulinresistenz und Hyperinsulinämie haben einen größeren Einfluss auf die Entstehung von Krebs- und Herz-KreislaufErkrankungen als der BMI. Die Weltgesundheitsorganisation benutzt deshalb den Bauchumfang von $80 \mathrm{~cm}$ für Frauen und von $94 \mathrm{~cm}$ für Männer als eine zuverlässigere Grenze zum Übergewicht; diese Werte entsprechen etwa einem BMI von 25.

Empfehlung 2:

Körperliche Aktivität sollte ein Teil des täglichen Lebens sein.

Mindestens 30 Minuten pro Tag sollte man moderat körperlich aktiv sein, vergleichbar mit schnellem Gehen. Für die Verbesserung der Leistungsfähigkeit sollten 60 Minuten moderate oder 30 Minuten intensive körperliche Aktivität angestrebt werden.

Die meisten Bevölkerungsgruppen, insbesondere solche, die in industrialisierter und städtischer Umgebung leben, sind körperlich weniger aktiv als sie es naturgemäß sein sollten. Körperliche Aktivität kann das Risiko für einige Krebsarten (Dickdarm, Brust, Gebärmutterschleimhaut, Prostata) mit einiger Wahrscheinlichkeit senken und beugt Übergewicht vor. Bestimmte Alltags-/Freizeitaktivitäten wie Gartenarbeit, Tanzen und handwerkliche Beschäftigungen können genauso wichtig sein (2) wie die üblichen sportlichen Aktivitäten Laufen, Fahrrad fahren und Schwimmen.

Empfehlung 3:

Der Verzehr energiedichter Lebensmittel sollte begrenzt werden.

Zuckerhaltige Getränke sind zu meiden. Fast Food sollte, wenn überhaupt, nur selten verzehrt werden. Der Konsum energiedichter Lebensmittel und gezuckerter Getränke nimmt derzeit weltweit zu und trägt vermutlich zum globalen Anstieg von Übergewicht bei. Energiedichte Lebensmittel (> $225 \mathrm{kcal} / 100 \mathrm{~g}$ ) verdrängen oft energiearme Lebensmittel (<125 kcal/100 g) mit hoher Nährstoffdichte. Gezuckerte Getränke tragen nur wenig zur Sättigung bei - hier liegt einer der Hauptgründe für Übergewicht, besonders bei Jugendlichen, die oft große Mengen dieser ungünstigen Durstlöscher konsumieren (13).

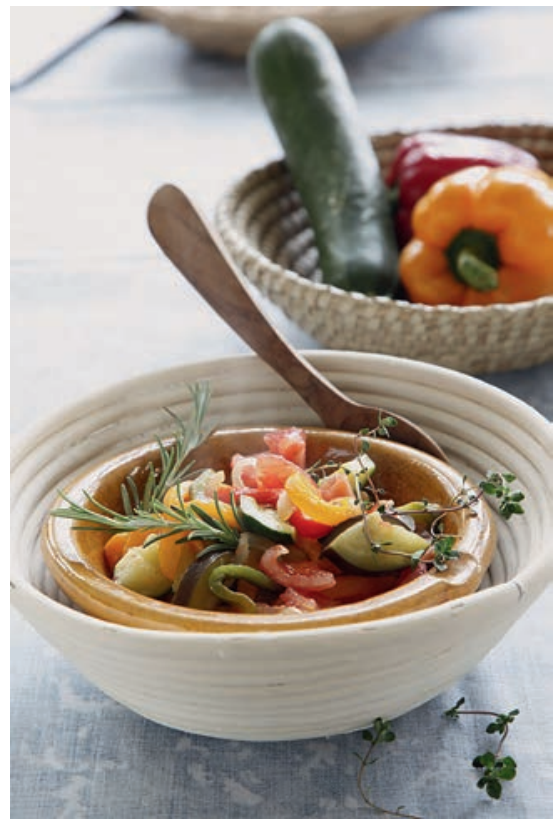

-Abb. 2 Prävention kann durchaus lecker sein: Einfach mehr Pflanzliches auf den Tisch. (c) Thieme Verlagsgruppe/Chris Meier

Empfehlung 4:

Überwiegend pflanzliche Lebensmittel verzehren.

Täglich sollten mindestens $400 \mathrm{~g}$ Gemüse und Obst sowie zu jeder Mahlzeit gering verarbeitetes Getreide und/oder Hülsenfrüchte verzehrt werden. Der Konsum von stark verarbeiteten, stärkehaltigen Lebensmitteln sollte begrenzt werden. Die vorliegenden Daten zeigen, dass die meisten Kostformen, die gegen Krebserkrankungen schützen, überwiegend aus pflanzlicher Nahrung bestehen ( $\vee$ Abb. 2).

Pflanzliche Lebensmittel wie Gemüse, Obst, Getreide, Hülsenfrüchte und Nüsse enthalten alle Nährstoffe, die sich auch in tierischen Lebensmitteln finden, ausgenommen Vitamin $B_{12}$ (9). Lakto-ovoVegetarier nehmen Vitamin $\mathrm{B}_{12}$ mit Milchprodukten und Ei auf, Veganer sind auf angereicherte Lebensmitteln oder Supplemente angewiesen (10). Ballaststoffe sowie eine große Anzahl antikanzerogener Verbindungen wie Carotinoide, Flavonoide und Glucosinolate, finden sich nur in pflanzlichen Lebensmitteln. 
Empfehlung 5:

Den Verzehr von rotem Fleisch

(Rind, Schwein, Schaf, Ziege)

begrenzen. Den Verzehr von

verarbeitetem Fleisch (gepökelt,

gebeizt, geräuchert) vermeiden.

Menschen, die regelmäßig Fleisch verzehren, sollten nicht mehr als $500 \mathrm{~g}$ pro Woche essen; davon sollte sehr wenig, wenn überhaupt, stark verarbeitet sein. Die Daten zeigen, dass viele Lebensmittel tierischer Herkunft einen wichtigen Beitrag zur Nährstoffversorgung leisten und gesundheitsförderlich sind, solange sie in mäßiger Menge verzehrt werden.

Ein hoher Verzehr von rotem Fleisch ist u.a. aufgrund des Hämeisengehalts mit einem erhöhten Risiko für Dickdarmkrebs verbunden. Der hohe Gehalt an Methionin im tierischen Protein (Fleisch, Milchprodukte) kann bei übermäßigem Verzehr problematisch sein (11). Eine Methioninrestriktion wird als eine mögliche Krebstherapie diskutiert und eine vegane Ernährung als Ernährungstherapie bei Krebs vorgeschlagen (4). Der Abbau von Methionin führt zur Bildung von toxischem Homocystein sowie Schwefelsäure, die eine Belastung für das Säure-Basen-Gleichgewicht darstellt.

Empfehlung 6:

Begrenzung des Konsums alkoholischer Getränke.

Der Konsum alkoholischer Getränke sollte auf nicht mehr als 2 Gläser pro Tag für Männer und ein Glas (10-15 g Alkohol) pro Tag für Frauen begrenzt werden. Alkoholkonsum ist mit einem erhöhten Krebsrisiko für Mund, Rachen, Speiseröhre, Dickdarm und Brust verbunden. Eigentlich sollte zur Krebsprävention kein Alkohol getrunken werden. Aber ein moderater Alkoholkonsum senkt wahrscheinlich das Risiko einer koronaren Herzkrankheit und verringert das Auftreten von Bluthochdruck, Diabetes und Krebs (Dickdarm, Eierstock und Prostata) (1). Eine gesunde Leber kann kleine Mengen an Alkohol problemlos abbauen, dennoch bleibt es ein allgemeines Zellgift, auch für das Gehirn.
Empfehlung 7:

Begrenzung des Salzkonsums. Der

Verzehr von verschimmelten

Getreide oder Hülsenfrüchten ist zu vermeiden.

Der Verzehr von gepökelten, gesalzenen oder salzigen Lebensmitteln ist zu vermeiden; Lebensmittel sollten ohne Salz haltbar gemacht werden. Ein hoher Salzkonsum kann das Magenkrebsrisiko u.a. durch eine Schädigung der Magenwand sowie Synergismen mit anderen Kanzerogenen erhöhen. Da ein hoher Salzkonsum bei salzsensitiven Menschen den Bluthochdruck fördert (5) und der größte Teil Salz mit vorgefertigten Lebensmitteln, besonders Fertigprodukte, Fast Food, Käse, Wurst und Brot, aufgenommen wird, sollte im Haushalt Salz sehr sparsam eingesetzt werden. Salzfreie Gewürze und Kräuter stellen gesunde Alternativen zu Salz dar (7).

Die Aufnahme von Aflatoxinen aus Schimmelpilzen steht im direkten $\mathrm{Zu}$ sammenhang mit Leberkrebs, der beson- ders in tropischen Ländern häufig zu finden ist.

Empfehlung 8:

Der Nährstoffbedarf sollte ausschließlich durch Lebensmittel gedeckt werden.

Nahrungsergänzungsmittel werden für die Krebsprävention nicht empfohlen. Ergebnisse mit hoch dosierten Supplementen können nicht auf die ganze Bevölkerung übertragen werden. Eine allgemeine Empfehlung, Nahrungsergänzungsmittel zur Prävention von Krebserkrankungen einzusetzen, könnte unerwartete und nachteilige Wirkungen mit sich bringen. Deshalb wird eine Erhöhung der Zufuhr relevanter Nährstoffe mittels der üblichen Kost vorgezogen.

Hoch dosierte Supplemente können die Nährstoffabsorption anderer Nährstoffe beeinflussen und das Krebsrisiko erhöhen (6). So kann hoch dosiertes, isoliertes Beta-Carotin als Supplement 
(nicht als Lebensmittel) das Lungenkrebsrisiko von Rauchern und eine zu hohe Kalziumzufuhr (über 1,5 g/Tag) wahrscheinlich das Prostatakrebsrisiko erhöhen.

Zwei Sonderempfehlungen gelten weltweit:

\section{Empfehlung 9: \\ Mütter sollten stillen; Säuglinge sollten gestillt werden.}

Säuglinge sollten möglichst bis zu 6 Monaten ausschließlich gestillt werden; danach sollte mit der Einführung von Beikost begonnen werden. Die wissenschaftlichen Daten für Krebs und andere Krankheiten zeigen, dass dauerhaftes, ausschließliches Stillen sowohl die Mutter als auch das Kind schützen ( $\vee$ Abb. 3 ). Stillen ist gut für Säugling und Mutter und es minimiert das Risiko, im späteren Lebensverlauf an Krebs zu erkranken (12).

Empfehlung 10:

\section{Für ehemalige Krebspatienten gelten die Empfehlungen zur Krebsprävention.}

Ehemals an Krebs Erkrankte sollten von ausgebildeten Ernährungsfachkräften betreut werden. Wenn möglich (und wenn es keine anderen Empfehlungen gibt), sollten die Empfehlungen für Ernährung, gesundes Körpergewicht und körperliche Aktivität eingehalten werden. Menschen, die von Krebs betroffen waren, befürchten eine Wiederkehr der Krankheit. Diese Angst selbst ist ein Risikofaktor, der durch gezielte Angstvermeidungsschulung verringert werden kann (8).

\section{Online}

http://dx.doi.org/10.1055/s-0034-1373892

\section{Literatur}

1 Arranz S, Chiva-Blanch G, Valderas-Martínez $P$ et al. Wine, beer, alcohol and polyphenols on cardiovascular disease and cancer. Nutrients 2012; 4: 759-781

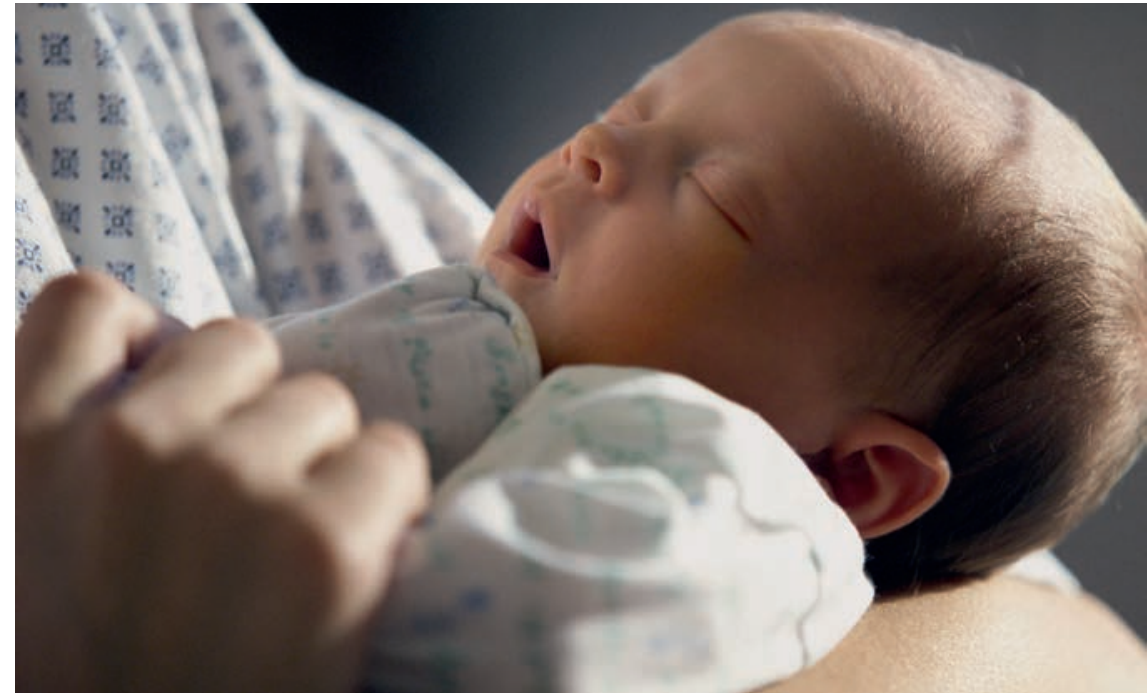

>Abb. 3 Prävention von Anfang an: Stillen hilft Kind und Mutter. @ PhotoDisc

2 Baumann F, Bloch W, Jäger E, Hrsg. Sport und körperliche Aktivität in der Onkologie. Heidelberg: Springer; 2012

3 Calori G, Lattuada G, Piemonti L. Prevalence, metabolic features and prognosis of metabolically healthy obese Italian individuals: the Cremona Study. Diabetes Care 2011; 34: 210-215

4 Cavouto $P$, Fenech $M$. A review of methionine dependency and the role of methionine restriction in cancer growth control and life-span extension. Cancer Treat Rev 2012; 38: $726-736$

5 Frisoli TM, Schmieder RE, Grodzicki T, Messerli FH. Salt and hypertension: is salt dietary reduction worth the effort? Am J Med 2012; 125: 433-439

6 Hart C, Cohen R, Norwood M, Stebbing J. The emerging harm of antioxidants in carcinogenesis. Future Oncol 2012; 8: $535-548$

7 Koerber K v, Männle T, Leitzmann C. Vollwert-Ernährung: Konzeption einer zeitgemäßen und nachhaltigen Ernährungsweise. 11. Aufl. Stuttgart: Haug; 2012

8 Lambert SD, Girgis A, Turner J et al. A pilot randomized controlled trial of the feasibility of a self-directed coping skills intervention for couples facing prostate cancer: rationale and design. Health Qual Life Outcomes 2012; 10: 119

9 Leitzmann C. Vegetarismus: Grundlagen, Vorteile, Risiken. 4. Aufl. München: Beck; 2012

10 Leitzmann C, Keller M. Vegetarische Ernährung. 3. Aufl. Stuttgart: Ulmer; 2013

11 López-Torres M, Barja G. Lowered methionine ingestion as responsible for the decrease in rodent mitochondrial oxidative stress in protein and dietary restriction possible implications for humans. Biochim Biophys Acta 2008; 1780: 1337-1347
12 Nagata C, Mizoue T, Tanaka K et al. Breastfeeding and breast cancer risk: an evaluation based on a systematic review of epidemiologic evidence among the Japanese population. Jpn J Clin Oncol 2012; 42: 124-130

13 Osei-Assibey G, Dick S, Macdiarmid J et al. The influence of the food environment on overweight and obesity in young children: a systematic review. BMJ Open 2012; 2: e001538

14 WCRF/AICR (World Cancer Research Fund/ American Institute for Cancer Research). Food, nutrition, physical activity, and the prevention of cancer: a global perspective. Washington DC: AICR; 2007

15 WCRF/AICR (World Cancer Research Fund/ American Institute for Cancer Research). Ernährung, körperliche Aktivität und Krebsprävention: Eine globale Perspektive. London: WCRF; 2007

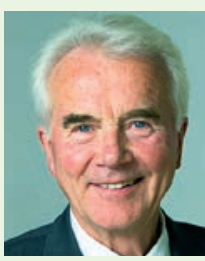

Prof. Dr. Claus Leitzmann Dörrenbergweg 24 35321 Laubach

Claus Leitzmann hat Chemie, Biochemie und Mikrobiologie in den USA studiert und war anschließend 5 Jahre als Dozent und Forscher in Thailand tätig, bevor er 1974 ans Institut für Ernährungswissenschaft der Universität Gießen kam. Neben der Vollwert-Ernährung befasst sich Prof. Leitzmann mit Ernährungsproblemen in Entwicklungsländern, Ernährungsbildung, vegetarischer Ernährung und Ernährungsökologie.

claus@leitzmann-giessen.de 